\title{
Influence of physical self and mindfulness on the academic possible selves of freshman nursing students
}

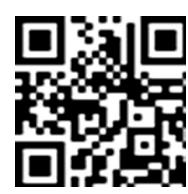

Original article

Mei-Ling Han, Hai-Ying Qu*

School of Humanities and Social Sciences, Binzhou Medical University, Yantai, Shandong 264003, China

Received: 26 February 2019; Accepted: 8 April 2019; Published: 20 September 2019

Abstract: Objective: The purposes of this study were to investigate the general level of the mindfulness, physical self, and academic possible selves in the freshman nursing students, and to explore the relationship among the three variables, so as to provide a theoretical basis for the freshmen to improve their academic achievement on the basis of the correct understanding of the physical self. Methods: A total of 360 freshman nursing students were enrolled in this study from a medical school in Yantai City. Data were collected using three questionnaires: Body Self-description Questionnaire, Five Factors Mindfulness Questionnaire, and Academic Possible Selves Questionnaire. There were 341 valid questionnaires.

Results: The mindfulness of the freshmen nursing students was at a medium level $(3.03 \pm 0.28)$, their attitude to physical self $(3.66 \pm 0.58)$ and academic possible selves $(3.33 \pm 0.50)$ were positive. There were significant gender differences among coordination, physical activity, athletic ability, strength, endurance, observation, non-response, total physical self-score, and negative future academic possible selves. In the above factors, except negative future academic possible selves, the scores for boys were higher than that for girls (all $P<0.05$ ). There were significant positive relationships between physical self, mindfulness, and academic possible selves. Self-esteem, acting with awareness, endurance, and description were effective positive predictors of the academic possible selves, with a total explanation rate of $33.6 \%$.

Conclusion: There were close relationships among the physical self, the level of mindfulness, and the academic possible selves. Positive physical self-description and high level of mindfulness could help individuals develop a positive attitude toward their academic possible selves.

Keywords: freshman nursing students $\bullet$ physical self $\bullet$ mindfulness $\bullet$ academic possible selves $\bullet$ medical school

(c) Shanxi Medical Periodical Press.

\section{Introduction}

The physical self is a basic and very important component of the whole self-concept, and it is the initial budding part of the individual's whole self-consciousness. It involves the cognitive evaluation of one's own appearance, athletic ability, health, and so on. The physical self is influenced by significant others and sociocultural environment, and further integrated into the overall selfconcept. ${ }^{1}$ Mindfulness is a complex multidimensional system, originated from the Buddhist Zen² thoughts in the East. So far, most researchers agree with KabatZinn's definition of mindfulness as a state of consciousness created by purposefully directing attention to the

How to cite this article: Han ML, Qu HY. Influence of physical self and mindfulness on the academic possible selves of freshman nursing students. Front Nurs. 2019; 3: 241-248. 
present. It is an individual's awareness of the various experiences that occur at this moment without judgment, in which the individual is more receptive to others and to himself. In 1999, Anderman ${ }^{3}$ defined the academic possible self as a cognitive representation of one's academic potential and future academic performance. As a future orientation component in the self-concept, possible self has two important functions, namely, the function of motivation and prediction, guiding and adjusting people's behavior. ${ }^{4}$

Relevant studies have shown that the overall level of health literacy among Chinese teenagers aged 15-24 years was low. ${ }^{5}$ The freshmen are at this age, as the promoters and popularizers of health education in future nursing work, they are required to have solid professional knowledge and skills, whereas the physical and mental health based on correct physical self-cognition is the cornerstone of their study and work, which will directly affect the level of nursing services in our country. ${ }^{6}$ Academic possible self was highly correlated with academic performance and academic behavior, which was a sensitive factor for predicting individual academic achievement and mental health. ${ }^{7}$ Wang ${ }^{8}$ research showed that the higher the academic possible self of junior middle school students, the better the academic performance, and the learning motivation played a partial intermediary role between the two. Qin Lin's ${ }^{9}$ research indicated that academic possible self could predict students' problematic behaviors to some extent. With the development of positive psychology, mindfulness training has become a widely used treatment in the field of psychology and education. Studies at home and abroad have shown that mindfulness intervention plays a strong role in regulating emotional processing and reducing procrastination. ${ }^{10-12}$

For freshmen, the academic role is still one of their main social roles. Academic possible self plays a very important role in the sound development of the individual's overall self. Academic possible self was not only an important manifestation of learning healthily for adolescents but also played an important role in maintaining and promoting their mental health. ${ }^{7}$ Therefore, for the freshman nursing students, academic possible self has important research value. At present, there are few studies on this area, and there is still a lack of research on the relationship among the physical self, the academic possible self, and the mindfulness of freshmen. This study explored the relationships among the three variables on the basis of understanding the physical self, the academic possible self, and the mindfulness of freshmen. From the perspective of positive psychology, this study suggests that there are significant positive relationships between physical self, mindfulness, and academic possible selves, and furthermore, the first two have a certain positive predictive effect on the latter. If these hypotheses are valid, nursing educators can help students promote positive learning behavior and improve academic achievement on the basis of correct understanding of their physical self.

\section{Subjects and methods}

\subsection{Subjects}

A stratified random sampling method was used to conduct a questionnaire survey on freshman nursing students in a medical college in Yantai city. A total of 360 questionnaires were distributed to participants who meet the following criteria: full-time student; freshman student at school; majoring in nursing (including three levels: junior college students, upgraded college students, and undergraduate students); and voluntary participation in this study. The researchers in this study included three uniformly trained graduate students majoring in psychology, and one of the researchers uniformly explained the purpose of the research and notes to the participants. After obtaining the informed consent of the class counselors, the investigation was carried out during the night classes, participants in this study were voluntary and anonymous. The questionnaires were uniformly provided, and the participants were allowed 30 minutes to answer all of questions about the three scales, and the questionnaires were collected immediately after completion. In all, 341 valid questionnaires were collected. The effective recovery rate was $94.7 \%$. Among them, there were 36 boys and 305 girls; 121 junior college students, 105 upgraded college students, and 115 undergraduate students.

\subsection{Research methods}

\subsubsection{Body Self-description Questionnaire}

The questionnaire was revised by Yang et al. ${ }^{13} \mathrm{~A}$ total of 70 items, included 11 dimensions: physical health, coordination, physical activity, obesity, athletic ability, the whole body, appearance, strength, flexibility, endurance, and body self-esteem, which rated on a six-point scale. The higher the score, the more positive the individual body self-description. The average score $<3.5$ means the body self-description is negative, the average score $=3.5$ means the body selfdescription is neutral, and the average score $>3.5$ means the body self-description is positive. Studies have shown that with good reliability and validity, the questionnaire is concise and easy to rate, and it has been widely used. 


\subsubsection{Five Factors Mindfulness Questionnaire}

The questionnaire was revised by Deng et al. ${ }^{14} \mathrm{~A}$ total of 39 items, included 5 factors: observing, describing, acting with awareness, non-judging, and non-reacting, which rated on a five-point scale. The higher the score, the higher the level of individual mindfulness. The average score $<3$ means lower level of mindfulness, the average score $=3$ means moderate level of mindfulness, and the average score $>3$ means higher level of mindfulness. The Cronbach's alpha of each dimension varied from 0.448 to 0.843 , the split-half reliability ranged from 0.439 to 0.843 , the test-retest reliability ranged from 0.436 to 0.741 , and the questionnaire has good calibration validity.

\subsubsection{Academic Possible Selves Questionnaire}

The questionnaire was revised by Wang et al. ${ }^{8} \mathrm{~A}$ total of 18 items, included 4 dimensions: positive present academic possible selves, positive future academic possible selves, negative present academic possible selves, and negative future academic possible selves, which rated on a five-point scale. The higher the score, the higher the level of academic possible selves, the average score $<3$ means negative attitude of academic possible selves, the average score $=3$ means neutral attitude of academic possible selves, and the average score $>3$ means positive attitude of academic possible selves. The questionnaire has good reliability and validity.

\subsection{Data analysis}

Data collection was conducted with a database built using Epidata 3.1. Descriptive statistics were used to examine the level of the mindfulness, physical self, and academic possible selves in the freshman nursing students. Independent t-test was performed to compare the mean scores of different genders. Correlations among the factors were analyzed by Pearson's correlation coefficient. Multiple regression analyses were used to examine the predictive effect factors on academic possible selves. Stepwise variable selection methods (probability of $F$, using 0.05 for entry and 0.10 for removal) were used to generate the multiple linear regression models. The variables considered for inclusion in each model included six factors of Body Self-description Questionnaire and two factors of Five Factors Mindfulness Questionnaire. All analyses were performed using SPSS 17.0. The significance level used in this analysis was $P<0.05$.

\section{Results}

\subsection{The general situation and sex difference of freshman nursing students' mindfulness, physical self, and academic possible selves}

The results showed that, on the whole, the mindfulness of the freshmen nursing students was at a middle level $(3.03 \pm 0.28)$, their attitude to physical self $(3.66 \pm 0.58)$, and academic possible selves were positive $(3.33 \pm 0.50)$. There were significant gender differences among coordination, physical activity, athletic ability, strength, endurance, observation, nonresponse, total physical self-score, and negative future academic possible selves. In the above factors, except negative future academic possible selves, the scores for boys were higher than that for girls (all $P<0.05$ ). There were no significant differences in other dimensions (see Table 1).

\subsection{Correlation analysis of physical self, level of mindfulness, and academic possible selves of freshman nursing students}

The results showed that there were significant positive relationships between physical self, mindfulness, and academic possible selves except for non-judging and non-reacting (see Table 2).

\subsection{Multiple linear regression analysis of physical self and level of mindfulness to academic possible selves of freshman nursing students}

Table 3 shows that the total score of academic possible selves of freshman nursing students was taken as the dependent variable, six dimensions of the physical self and two dimensions of the mindfulness were used as independent variable, and then the influence of independent variables on dependent variable was explored by stepwise regression analysis. Four regression models were obtained by analysis, and the values of $R, R^{2}$, and $\Delta R^{2}$ of the fourth model were optimal. Therefore, the standard regression equation was selected in this optimal model: Academic possible self $=21.626+0.556$ body self-esteem +0.268 acting with awareness +0.201 endurance +0.245 description. From this equation, body self-esteem, acting with awareness, endurance, and description can effectively predict the academic possible self of freshman nursing students, and the total explanation rate of these five factors is $33.6 \%$. 


\begin{tabular}{|c|c|c|c|c|}
\hline Project & Totality & Male & Female & $t$ \\
\hline Physical health & $4.48 \pm 0.62$ & $36.36 \pm 5.19$ & $35.75 \pm 4.89$ & 0.71 \\
\hline Coordination & $3.45 \pm 0.89$ & $22.39 \pm 7.08$ & $20.51 \pm 5.09$ & $2.01^{*}$ \\
\hline Physical activity & $2.68 \pm 0.74$ & $19.14 \pm 4.87$ & $15.69 \pm 4.26$ & $4.52^{\star *}$ \\
\hline Obesity & $3.70 \pm 1.22$ & $22.00 \pm 9.30$ & $22.22 \pm 7.04$ & -0.17 \\
\hline Athletic ability & $3.15 \pm 0.99$ & $20.81 \pm 8.03$ & $18.66 \pm 5.66$ & $2.05^{\star}$ \\
\hline The whole body & $3.51 \pm 0.91$ & $21.06 \pm 7.75$ & $21.04 \pm 5.13$ & 0.02 \\
\hline Appearance & $3.95 \pm 0.93$ & $23.39 \pm 6.88$ & $23.70 \pm 5.43$ & -0.32 \\
\hline Strength & $3.36 \pm 0.85$ & $22.28 \pm 6.99$ & $19.89 \pm 4.76$ & $2.69 * *$ \\
\hline Flexibility & $3.53 \pm 0.85$ & $22.36 \pm 6.54$ & $21.02 \pm 4.92$ & 1.50 \\
\hline Endurance & $3.24 \pm 1.05$ & $22.31 \pm 7.94$ & $19.11 \pm 5.97$ & $2.93^{* *}$ \\
\hline Body self-esteem & $4.63 \pm 0.73$ & $36.99 \pm 7.64$ & $37.06 \pm 5.59$ & -0.07 \\
\hline Physical self & $3.66 \pm 0.58$ & $269.08 \pm 59.45$ & $254.64 \pm 37.79$ & $2.02^{*}$ \\
\hline Observing & $2.76 \pm 0.59$ & $23.64 \pm 4.91$ & $21.87 \pm 4.74$ & $2.11^{\star}$ \\
\hline Describing & $3.13 \pm 0.59$ & $24.69 \pm 6.19$ & $25.06 \pm 4.61$ & -0.44 \\
\hline Acting with awareness & $3.60 \pm 0.68$ & $27.22 \pm 5.26$ & $28.97 \pm 5.40$ & -1.84 \\
\hline Non-judging & $2.95 \pm 0.58$ & $22.67 \pm 4.61$ & $23.71 \pm 4.67$ & -1.27 \\
\hline Non-reacting & $2.66 \pm 0.52$ & $20.75 \pm 4.36$ & $18.36 \pm 3.49$ & $3.79^{* *}$ \\
\hline Level of mindfulness & $3.03 \pm 0.28$ & $118.97 \pm 11.96$ & $117.97 \pm 10.59$ & 0.53 \\
\hline Positive present academic possible selves & $3.11 \pm 0.55$ & $15.56 \pm 4.61$ & $15.93 \pm 3.56$ & -0.57 \\
\hline Negative present academic possible selves & $3.55 \pm 0.55$ & $11.50 \pm 2.41$ & $12.16 \pm 2.02$ & -1.82 \\
\hline Positive future academic possible selves & $2.65 \pm 0.61$ & $19.56 \pm 4.92$ & $19.29 \pm 4.09$ & 0.36 \\
\hline Negative future academic possible selves & $4.03 \pm 0.69$ & $12.06 \pm 1.99$ & $12.73 \pm 1.73$ & $-2.17^{\star}$ \\
\hline Academic possible selves & $3.22 \pm 0.70$ & $58.67 \pm 11.65$ & $60.11 \pm 8.73$ & -0.90 \\
\hline
\end{tabular}

Note: ${ }^{*} P<0.05,{ }^{\star} * P<0.01$.

Table 1. The general situation and sex difference of freshman nursing students' mindfulness, physical self, and academic possible selves $(\mathrm{M} \pm \mathrm{SD})$

\section{Discussion}

\subsection{Physical self-condition of freshman nursing students}

On the whole, the freshman nursing students showed a positive attitude toward the body self-description. They held relatively positive body self-cognition in six dimensions of body self-esteem, physical health, appearance, obesity, flexibility, and the whole body, while holding relatively negative body self-cognition in five dimensions of coordination, strength, endurance, athletic ability, and physical activity, it should be noted that the description of physical activity was the most negative. To some extent, this showed that the freshman nursing students had less physical activity. With the deepening of the research on health connotation, the fitness value of physical activity has been increasingly concerned by the society. Having a healthy body is the basic material prerequisite for medical students to do well in medical work in the future, it also helps to better guide patients to prevent disease and promote health through a good combination of medical treatment and physical exercise. ${ }^{15}$ Studies have shown that the adverse factors affecting medical students' participation in sports activities include heavy learning tasks, imperfect school site facilities and equipment, lack of basic knowledge and skills of sports, weak motivation and awareness of sports activities. ${ }^{16}$ Therefore, the school should coordinate the individual needs of students and the requirements of social development on students' physical education, stand on the height of educating people, standardize the formation of students' sports values, and strive to improve the reform of teaching methods and the equipment of sports facilities. Students themselves should improve their exercise consciousness, develop good habit of physical exercise, arrange their study, and exercise time reasonably, so as to actively participate in daily study and life with a healthier state of mind and body. ${ }^{17}$

In terms of gender, boys' descriptions of coordination, physical activity, athletic ability, strength, and endurance as well as their overall physical selves were all significantly more positive than girls, which were consistent with previous studies. ${ }^{18}$ This reflects that the enthusiasm of boys to participate in sports activities is 


\begin{tabular}{lcccccc}
\hline $\begin{array}{l}\text { Independent } \\
\text { variable }\end{array}$ & $R$ & $R^{2}$ & $\begin{array}{c}\text { Adjusted } \\
R^{2}\end{array}$ & $B$ & $\beta$ & $t$ \\
\hline \hline $\begin{array}{l}\text { Constant } \\
\text { term }\end{array}$ & 0.586 & 0.344 & 0.336 & 21.626 & & $7.321^{* *}$ \\
$\begin{array}{l}\text { Body self- } \\
\text { esteem }\end{array}$ & & & & 0.556 & 0.357 & $6.487^{* *}$ \\
$\begin{array}{l}\text { Acting with } \\
\text { awareness }\end{array}$ & & & & & & \\
Endurance & & & 0.268 & 0.160 & $3.196^{* *}$ \\
Description & & & 0.201 & 0.139 & $2.953^{* *}$ \\
& & & 0.245 & 0.129 & $2.510^{*}$ \\
\hline
\end{tabular}

Note: ${ }^{*} P<0.05,{ }^{*} P<0.01$

Table 3. Multiple linear regression analysis of physical self and mindfulness to academic possible selves of freshman nursing students

higher than that of girls, which, in turn, makes boys' athletic ability more powerful, and their corresponding abilities in coordination, strength, and endurance are also higher than that of girls. ${ }^{19}$ Gender is the basic component of self-concept. Gardner et al. ${ }^{20}$ showed that gender cultural stereotypes also have an effect on the physical self-description of both sexes. Therefore, in the process of physical education, we should pay attention to the influence of the gender role and subjective tendency of college students on their physical exercise behavior orientation, combining with the characteristics of gender roles to teach students according to their aptitude. ${ }^{15}$ There was no significant difference between the two sexes in other dimensions of physical self, that is, the description and evaluation of physical health, obesity, the whole body, appearance, flexibility, and selfesteem were not affected by gender factors. This was not consistent with the results of Sun Yinjia, ${ }^{18}$ who used the same scale. By analyzing the reasons, her subjects were junior high school students, and the gender difference in puberty may lead to differences in the attention of male and female students to physical obesity, appearance, and health. This showed from the side that cognitive differences in certain aspects of the sexes may decrease with age.

\subsection{The level of mindfulness of freshmen}

On the whole, the mindfulness of the freshmen was at the middle level, which supported the research of Wang et al. ${ }^{21}$ Among them, the levels of acting with awareness and description were high, and the levels of nonjudgment, observation, and non-reacting were low. This showed that, at present, the freshmen could clearly describe, understand, and pay attention to their current behavior, but could not deal with internal and external stimuli in an objective way, and thus could not obtain more objective information in the observation without judgment, which was not conducive to them to deal with 
the problem from a broader and flexible perspective. In general, there was no significant difference in the level of mindfulness between the sexes, which was consistent with the previous studies at home and abroad, ${ }^{22-24}$ indicating that gender was not a factor influencing the level of mindfulness. In terms of observation and nonreacting, the level of boys was significantly higher than that of girls, that is, boys could maintain a more objective and comprehensive understanding of the internal and external stimuli acting on themselves, and were not anxious to react immediately under the control of habitual emotions and thoughts, which was beneficial to solve the problem from multiple angles. ${ }^{25}$

\subsection{The academic possible selves of freshman nursing students}

On the whole, the freshman nursing students' academic possible selves were little higher than medium. Specifically, except for the positive proximal academic possible self, which was at a lower level, the academic possible selves in other dimensions were above the middle level, and distal academic possible self was more positive than the proximal academic possible self. This showed that, on the whole, the freshman nursing students had a positive attitude toward their academic possible self. With the growth of age, college students' self-awareness and self-evaluation ability are gradually improved, under the influence of multiple factors such as new environment's competition consciousness and parents' expectation, their self-expectation in academic performance will be more positive. ${ }^{26}$ In terms of positive proximal academic possible self, most freshmen have not yet fully adapted to the changes in learning environment and teaching methods of the university campus. The frustration caused by maladaptation may, to some extent, reduced their positive attitude toward their academic ability at the current stage. In addition, as this study adopted the questionnaire survey method, participants might be more modest in their assessment of their current academic possible self. For students who were looking forward to the future, they generally did not have very positive feelings and perceptions about their current academic performance. ${ }^{27}$

In terms of gender, boys had significantly lower scores in negative distal academic possible selves than girls, that is, compared with girls, boys hold a more positive attitude toward future studies, which was inconsistent with Wang's research. ${ }^{8}$ Analyzing the reasons, although nursing departments are dominated by female students, with the development of modern nursing career, to adapt to the increasing clinical demand for male nurses, relevant colleges and universities gradually optimize the teaching mode, pay more attention to the psychological characteristics of male nursing students and teach them according to their aptitude. ${ }^{28} \mathrm{An}$ increasing number of male nursing students realized that nursing has a better development future and are more confident about their future career. ${ }^{29} \mathrm{~A}$ higher level of professional identity could reduce the waste of teaching resources and brain drain. ${ }^{30}$ Therefore, while changing the influence of external environment on male nursing students, we should also actively strengthen their professional ideological education. It was worth noting that Duffy's research showed that negative possible self was more predictive than positive possible self, ${ }^{31}$ so educators should pay enough attention to this aspect.

\subsection{The relationship among physical self, level of mindfulness, and academic possible selves of freshman nursing students}

This study showed that there was a significant positive correlation among physical self, level of mindfulness, and academic possible selves. In the further regression analysis, self-esteem, acting with awareness, endurance, and description could effectively predicted the academic possible selves of freshman nursing students, and the total explanation rate of these four factors was $33.6 \%$. Studies have shown that body image is associated with many dimensions of mindfulness. Mindfulness could increase the individual's non-judgment on the body, confirm to the observation of internal feelings and reduce the destructive behavior to the body when the individual had negative body image, that is, the higher the level of mindfulness, the higher the physical selfsatisfaction. ${ }^{32}$ For freshmen, physical self and academic self were indispensable parts of their self-concept structure and specific expressions of self-concept in different fields. ${ }^{20}$ Numerous studies have shown that there is a close relationship between physical self and academic possible selves. ${ }^{19}$ Positive physical self-description was a favorable factor for the academic possible selves of freshmen. Pajas and Schunk pointed out that in the educational environment, students' perception of their academic ability is a key factor affecting their academic success. When experiencing some negative emotions of the physical self, people with a high level of mindfulness could calmly and objectively observe and describe the internal and external stimuli acting on them, so as to form a relatively objective understanding of themselves. On the basis of a clearer understanding of the external environment and internal response patterns, correctly identified which reactions were based on realities and which were derived from past experiences and patterns; thus, there was more freedom to choose how to respond to realistic stimuli. ${ }^{33}$ 
The physical characteristics of an individual were objective, but the perception of the body was subjective and changeable, blindly comparing with the ideal body model in culture would inevitably hurt the sense of self-worth associated with the body and thus affect other aspects of learning and life. ${ }^{34}$ College period is an important node for students to step into society and cultivate consciousness of lifelong physical exercise. ${ }^{35}$ As the reserve force of nursing talents, the physical quality and physical exercise concept of nursing students in medical colleges will have an important impact on their future mental health, professional development and other aspects. Therefore, the society, colleges, and other relevant departments should pay more attention to the current situation of physical exercise of nursing students, through the improvement of teaching method reform and sports facilities, mindfulness training, and other ways to guide nursing students to improve their negative physical cognition on the basis of scientific exercise, so as to achieve the purpose of improving overall self-esteem and academic achievement. ${ }^{18}$

\section{Conclusions}

On the whole, the mindfulness of the freshmen nursing students was at a medium level, their attitude to physical self and academic possible selves were positive. There were close relationships between the physical self, the level of mindfulness, and the academic possible selves. Positive physical self-description and high level of mindfulness could help individuals develop a positive attitude toward their academic possible selves.

Nursing educators can help students promote positive learning behavior and improve academic achievement on the basis of correct understanding of their physical self. For example, the school should coordinate the individual needs of students and the requirements of social development on students' physical education, strive to improve the reform of teaching methods and the equipment of sports facilities, pay more attention to the psychological characteristics of male nursing students, and teach them according to their aptitude. Students themselves should improve their exercise consciousness, develop good habit of physical exercise, arrange their study and exercise time reasonably, so as to actively participate in daily study and life with a healthier state of mind and body.

\section{Limitations and Advantages}

First of all, because of the small sampling of this research limited in one university in Yantai, the research results are still explorative but not qualified to be popularized. Second, as this study is a cross-sectional study, it is difficult to obtain the continuous psychological change process.

For freshmen, the academic role is still one of their main social roles. Academic possible self plays a very important role in the sound development of the individual's overall self. At present, there are few studies on the academic possible self, and there is still a lack of research on the relationship among the physical self, the academic possible self, and the level of mindfulness of freshmen. This study explored the relationships among the three variables on the basis of understanding the physical self, the academic possible self, and the mindfulness of freshmen. The results provided a theoretical basis for the freshman to promote positive learning behavior and improve academic achievement on the basis of correct understanding of their physical self.

\section{Ethics approval}

This study met the ethical requirements of the Helsinki Declaration. After obtaining the informed consent of the class counselors, the investigation was carried out during the night classes, and participants in this study were voluntary and anonymous.

\section{Conflicts of interest}

All contributing authors declare no conflicts of interest.

\section{References}

1. Li MC. The Relationship Between Physical Self and Physical Exercise Behavior of College Students. Nanjing Normal University; 2014 (in Chinese).

2. Jon K-Z. Mindfulness-based interventions in context: past, present, and future. Clin Psychol Sci Pract. 2003;10:144-156.

3. Anderman EM, Anderman LH, Griesinger T. The relation of present and possible selves during early adolescence to grade point average and achievement goals. Elem Sch J. 1999;100:3-17.

4. Li J. The Relationship Between High School Students' Time Comparison and Academic Possible Selves. Shenyang Normal University; 2013 (in Chinese).

5. Wei W, Li YH, Li FB. Analysis on the current situation of health literacy of residents aged 15-24 in 
China. Chin J Health Educ. 2015;31:104-107 (in Chinese).

6. Li J, Sun XQ. Survey on the current mental health of 272 undergraduate nursing students. China $J$ Health Psychol. 2017;25:754-757 (in Chinese).

7. Guo C. Academic Self-Study of Adolescents. Southwest University; 2006 (in Chinese).

8. Wang CJ. The Influence of Junior High School Students' Academic Possible Self on their Academic Achievement - The Mediating Role of Learning Motivation. Henan University; 2011 (in Chinese).

9. Qin L. Academic Possible Self and Problem Behavior of Junior High School Students. Capital Normal University; 2007 (in Chinese).

10. Liu L, Wang HF, Chen CY. Effects of mindfulness meditation training level on emotional processing. Psychol Sci. 2016;39:1519-1524 (in Chinese).

11. He YQ, Wang LF, Liu Y. Effects of mindfulness group training on procrastination of college students. Chin Health Serv Manage. 2017;34:546-549 (in Chinese).

12. Roemer LH, Williston SK, Rollins LG. Mindfulness and emotion regulation. Curr Opin Psychol. 2015;3:52-57.

13. Yang J. Introduction and revision of PSDQ. Shandong Sports Sci Technol. 2002;24:83-86 (in Chinese).

14. Deng $Y Q$, Liu $X H$, Rodriguze MA, Xia CY. The Five facet mindfulness questionnaire: psychometric properties of the Chinese version. Mindfulness. 2011;2:123-128.

15. Wang GN. Research on the Present Situation and Countermeasures of Sports Activities of Medical College Students in Beijing. Beijing Sports University; 2014 (in Chinese).

16. Mo Z, Tang ZL, Li Y. Investigation on the current situation of extracurricular physical exercise of medical school in Dali University. Chin J Sch Doc. 2011;25:310-312 (in Chinese).

17. Zhang ZM, Chen N. The realistic appeal and theoretical orientation of the transformation in the mode of school sports development in China. J Beijing Sport Univ. 2015;38:88-94 (in Chinese).

18. Sun YJ. Study on the Current Situation of Physical Self-Concept of Middle School Students and its Correlation with Academic Possible Self. Suzhou University; 2016 (in Chinese).

19. Huo Q. Urban-Rural and Gender Differences in the Relationship Among Adolescents' Physical Self, Academic Self and Life Satisfaction. Beijing Sport University; 2008 (in Chinese).

20. Gardner RM, Sorter RG, Friedman BN. Developmental changes in children's body images. J Soc Behav Pers. 1997;12:1019-1036.

21. Wang RM, Shang QY, Zhao S, et al. Study on the influence of nursing undergraduates' mindfulness level on internship stress. Chin J Nurs Educ. 2017;14:129-133 (in Chinese).

22. MacKillop J, Anderson EJ. Further psychometric validation of the Mindful Attention Awareness Scale (MASS). J Psychopathol Behav Assess. 2007;29:289-293.

23. Alexander V, Tatum C. Gender differences in anxiety in a mindfulness and cognitive therapy treatment program follow up. J Mens Health. 2010;7:330-331.

24. Yang YX. Impact of Mindfulness Training to University Students' Emotional Self-Efficacy and Subjective Well-Being. Shenyang Normal University; 2014 (in Chinese).

25. Meng Y. The Relationship Between Rumination and Mindfulness Self-Concern: The Moderating Effect of Prosocial Behavior. Qufu Normal University; 2015 (in Chinese).

26. Xie Y. Analysis of the present situation of academic possible self in middle school students. Guide Sci Educ. 2014;5:227-229 (in Chinese).

27. Chen SM, Qi JY, Ni QQ, Zhou F, Xu HB. Study on the the current situation of male nursing students' inferiority complex and its relationship with personality characteristics and social support. Nurs Rehabil J. 2016;15:423-427 (in Chinese).

28. Li P, Li ZL, Qin Q, Guo B, He WB. Exploration and practice of training mode of male nursing students. Chin Gen Pract Nurs. 2017;15:3052-3054 (in Chinese).

29. Jiang $X Y$, Xiao HM, Zhang $X$. Analysis and countermeasures of influencing factors on professional attitude of nursing students. Chin J Nurs Educ. 2007:4:3-7 (in Chinese).

30. Zhu ZL, Zhong X. Qualitative study on professional identity of male nursing students in colleges and universities. Today Nurse. 2011:4:160-162 (in Chinese).

31. Duffy K. Self-conception and self development. Psychol Life. 2006;6:133-121.

32. Stewart T. Light on body image: Acceptance through mindfulness. Behav Modif. 2004;28:783-811.

33. Zhu Y, Wang HY. Analysis on the relationship between female college students' physical self and mindfulness. Chin J Sch Health. 2012;33:735-736 (in Chinese).

34. Sun YL. Study on the Influence of Adolescents Negative Physical Self on their SELF-Esteem and Academic Achievement. Jiangxi Normal University; 2009 (in Chinese).

35. Wang J, Dong CC, Zhao PH. Analysis on the influencing factors of physical exercise for college students majoring in nursing. Sci Technol Inf. 2015;13:214, 216 (in Chinese). 